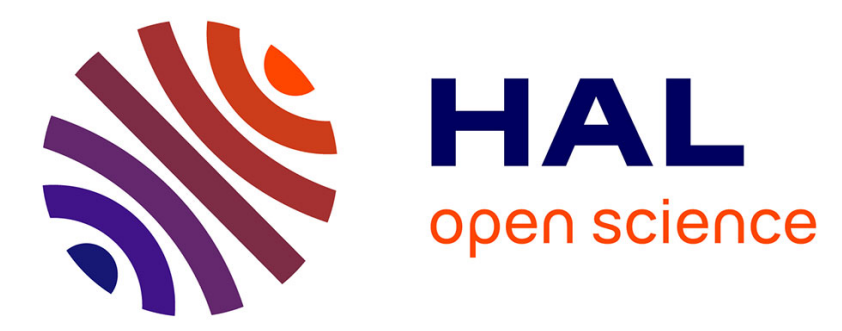

\title{
Quantum-nondemolition measurements using ghost transitions
}

Klaus M. Gheri, Philippe Grangier, Jean-Philippe Poizat, Daniel F. Walls

\section{To cite this version:}

Klaus M. Gheri, Philippe Grangier, Jean-Philippe Poizat, Daniel F. Walls. Quantum-nondemolition measurements using ghost transitions. Physical Review A : Atomic, molecular, and optical physics [1990-2015], 1992, 46, pp.4276-4285. 10.1103/PhysRevA.46.4276 . hal-00559005

\section{HAL Id: hal-00559005 \\ https://hal-iogs.archives-ouvertes.fr/hal-00559005}

Submitted on 23 May 2016

HAL is a multi-disciplinary open access archive for the deposit and dissemination of scientific research documents, whether they are published or not. The documents may come from teaching and research institutions in France or abroad, or from public or private research centers.
L'archive ouverte pluridisciplinaire HAL, est destinée au dépôt et à la diffusion de documents scientifiques de niveau recherche, publiés ou non, émanant des établissements d'enseignement et de recherche français ou étrangers, des laboratoires publics ou privés. 


\title{
Quantum-nondemolition measurements using ghost transitions
}

\author{
Klaus M. Gheri \\ Physics Department, University of Auckland, Private Bag 92019, Auckland, New Zealand \\ Philippe Grangier and Jean-Philippe Poizat \\ Institut d'Optique, Bô̂te Postale 147, 91403 Orsay, France \\ Daniel F. Walls* \\ Joint Institute for Laboratory Astrophysics, University of Colorado, Boulder, Colorado 80309-0440
}

(Received 10 March 1992)

\begin{abstract}
We present a detailed analysis of the quantum-nondemolition (QND) properties of a measurement scheme employing coherently driven three-level atoms in the $\Lambda$ or cascade configuration inside an optical cavity. We propose to use a strong signal field to empty one of the transitions and dress the signaltransition levels. The atoms become transparent for the signal beam, which sees a "ghost transition." When a probe is applied and tuned to the vicinity of one of the light-shifted levels, the probe phase is extremely sensitive to fluctuations of the signal intensity. This enhances the QND coupling and enables the system to operate at lower cooperativities.
\end{abstract}

PACS number(s): 42.50.Lc, 42.50.Dv

\section{INTRODUCTION}

A quantum-nondemolition (QND) measurement may be implemented in optics via a four-wave-mixing interaction [1-3]. In such systems a measurement of the phase fluctuations of a probe field enables the amplitude fluctuations to be determined in a manner which evades back-action noise. Experiments demonstrating this effect have been performed by Levenson et al. [4] using fourwave mixing in optical fibers and by Grangier, Roch, and Roger [5], who coupled two electromagnetic-field modes via a two-photon cascade transition in a three-level atom. The QND correlations obtained in these experiments have been limited by excess noise.

In the experiment of Grangier, Roch, and Roger [5], the signal and probe beams were significantly detuned from the intermediate level to avoid absorption and spontaneous-emission noise. In order to compensate for the loss of interaction strength, it was necessary to increase the density of atoms. Increasing the density of atoms, however, increases noise due to collisions. We seek a way to obtain a large QND gain with smaller atomic cooperativities. Several theoretical analyses [6-8] of QND measurements using three-level atoms in the cascade configuration have been made, the most complete being that of Poizat, Collett, and Walls [8], where the intermediate level is included.

In this paper we wish to describe a proposal where one atomic transition is coherently driven by a strong signal field and the remaining transition is scanned by a much weaker probe beam. The atoms may be in the $\Lambda$ or cascade configuration. In the latter case, the signal drives the upper transition [9] [cf. Figs. 1(a) and 1(b)]. The effect of the strong pumping by the signal is that almost all the population will be in the ground state of the probed atomic transition. Thus the signal will be applied to an essentially empty transition ("ghost transition"), which greatly reduces signal absorption and spontaneous-emission noise. Since the atomic medium is effectively transparent for the signal, degradation is negligible.

The strong signal field will dress the atomic levels be-
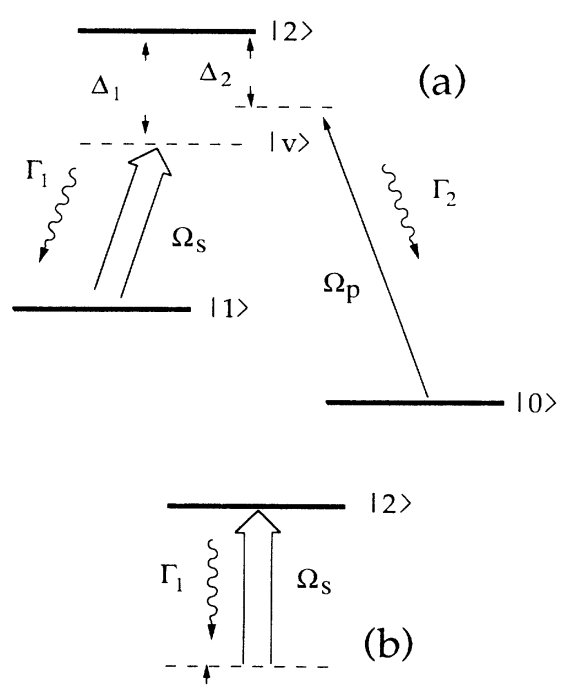

$\Delta_{1}$

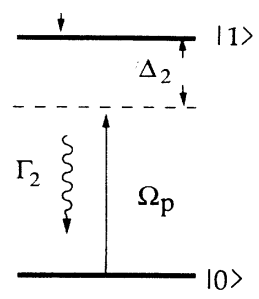

FIG. 1. (a) $\Lambda$-model atom. (b) Cascade-model atom. 


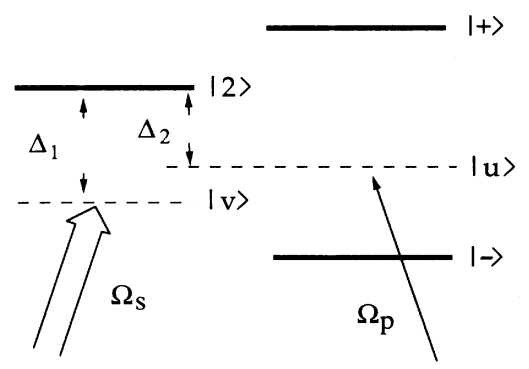

FIG. 2. Strong signal field $\Omega_{s}$ dresses the signal transition and creates the dressed states $|+\rangle,|-\rangle$. Tuning the probe $\Omega_{p}$ to the virtual level $|v\rangle$ corresponds to the two-photonresonance case for which, in the case of $\Lambda$ atoms, the atoms become transparent to probe and signal beam due to coherent population trapping.

tween which it is applied. The strength of the correlation between signal and probe may be increased by tuning the probe to the vicinity of one of the Rabi-split levels (Fig. 2 ), while still being outside the absorption line. Thus the probe phase will be highly sensitive to any changes in the signal intensity.

The organization of this paper is as follows. We shall first give in Sec. II a presentation of the criteria characterizing nonideal QND measurements. We present then in Sec. III analytical approximated results for the $\Lambda$ configuration, which will allow us to get a first insight into the QND coupling between the signal and probe beams. In Sec. IV we discuss these results by comparing them with numerical solutions of the complete model (see below). In Sec. $\mathrm{V}$ we shall deal with the cascade configuration and compare it with the $\Lambda$ scheme and with the results of Ref. [8]. We present in the last section (Sec. VI) the mathematical model used for the numerical computations.

\section{QND CRITERIA}

The aim of an optical QND measurement is to measure a quadrature component of a signal beam without perturbing it by coupling it to a quadrature of a probe (or meter) beam. The effectiveness of such a measurement can be characterized by three criteria proposed by Holland et al. [12] using correlation coefficients. These criteria have been recently reconsidered by Grangier, Courty, and Reynaud [13] under the scope of equivalent input noise, which makes them better suited for an experimental characterization via classical transfer measurements.

The first of these criteria is that a measurement of the signal is actually performed, i.e., that the meter beam has picked up some information about the signal. This can be done by considering the normalized correlation between the input signal and output of the meter beam. This coefficient is defined the following way:

$$
C^{2}\left(X_{s}^{\text {in }} Y_{p}^{\text {out }}\right)=\frac{\left|\left\langle X_{s}^{\text {in }} Y_{p}^{\text {out }}\right\rangle_{\text {sym }}\right|^{2}}{\left\langle\left(X_{s}^{\text {in }}\right)^{2}\right\rangle\left\langle\left(Y_{p}^{\text {out }}\right)^{2}\right\rangle},
$$

where $X_{s}$ and $Y_{p}$ are, respectively, the signal and probe quadrature operators defined further in Eq. (10) and where

$$
\left\langle X_{s}^{\text {in }} Y_{p}^{\text {out }}\right\rangle_{\text {sym }}=\frac{1}{2}\left(\left\langle X_{s}^{\text {in }} Y_{p}^{\text {out }}\right\rangle+\left\langle Y_{p}^{\text {out }} X_{s}^{\text {in }}\right\rangle\right) .
$$

We have $C^{2}\left(X_{s}^{\text {in }} Y_{p}^{\text {out }}\right) \in[0,1]$. For a perfect measurement, we have $C^{2}\left(X_{s}^{\text {in }} Y_{p}^{\text {out }}\right)=1$. For coherent inputs the corresponding equivalent input noise is related to this coefficient by (see Ref. [13] for more details)

$$
C^{2}\left(X_{s}^{\text {in }} Y_{p}^{\text {out }}\right)=\frac{1}{1+N^{\text {eq }}\left(X_{s}^{\text {in }} Y_{p}^{\text {out }}\right)} .
$$

For a perfect measurement, we have $N^{\mathrm{eq}}\left(X_{s}^{\text {in }} Y_{p}^{\text {out }}\right)=0$.

The second criterion characterizes the signalnondemolition property of the device. The correlation coefficient that will quantify this property is given by

$$
C^{2}\left(X_{s}^{\text {in }} X_{s}^{\text {out }}\right)=\frac{\left|\left\langle X_{s}^{\text {in }} X_{s}^{\text {out }}\right\rangle_{\text {sym }}\right|^{2}}{\left\langle\left(X_{s}^{\text {in }}\right)^{2}\right\rangle\left\langle\left(X_{s}^{\text {out }}\right)^{2}\right\rangle} .
$$

We have $C^{2}\left(X_{s}^{\text {in }} X_{s}^{\text {out }}\right) \in[0,1]$. For a perfectly nondegrading device, we have $C^{2}\left(X_{s}^{\text {in }} Y_{p}^{\text {out }}\right)=1$.

The third criterion tells us how well the probe output is correlated with the signal output. A convenient quantity for characterizing it is the variance in the signal output, given a measured value of the probe field (conditional variance). We first define the correlation coefficient

$$
C^{2}\left(X_{s}^{\text {out }} Y_{p}^{\text {out }}\right)=\frac{\left|\left\langle X_{s}^{\text {out }} Y_{p}^{\text {out }}\right\rangle_{\text {sym }}\right|^{2}}{\left\langle\left(X_{s}^{\text {out }}\right)^{2}\right\rangle\left\langle\left(Y_{p}^{\text {out }}\right)^{2}\right\rangle} .
$$

The conditional variance can then be written (for a linearized system) as

$$
V\left(X_{s}^{\text {out }} \mid Y_{p}^{\text {out }}\right)=\left\langle\left(X_{s}^{\text {out }}\right)^{2}\right\rangle\left[1-C^{2}\left(X_{s}^{\text {out }} Y_{p}^{\text {out }}\right)\right] .
$$

This criterion gives in fact the state-preparation ability of the system. For a perfect state-preparation device $V\left(X_{s}^{\text {out }} \mid Y_{p}^{\text {out }}\right)=0$, whereas $V\left(X_{s}^{\text {out }} \mid Y_{p}^{\text {out }}\right)=1$ (no quantum effect) for a beam splitter.

Note that the ordering has to be considered very carefully when dealing with frequency spectra of these various coefficients (see Refs. $[8,13]$ ).

\section{III. $\Lambda$ CONFIGURATION-ANALYTICAL RESULTS}

In this first part we want to give an account of the interaction of three-level atoms in the $\Lambda$ configuration with two coherent light fields as depicted in Fig. 1(a). We assume the light fields to be detuned from the atomic resonances by an amount of $\Delta_{1}$ and $\Delta_{2}$, respectively. The spontaneous decay rates are denoted by $2 \Gamma_{1}$ and $2 \Gamma_{2}$. The decay rate for both atomic coherences is then $\gamma=\Gamma_{1}+\Gamma_{2}$. Furthermore, we assume that the Rabi frequency $\Omega_{s}$ of beam 1 , from now on called the signal beam, is much stronger than the Rabi frequency $\Omega_{p}$ of beam 2 , or the probe beam. The immediate consequence of this assumption is that in a first approximation we may neglect the effect of the probe beam on the atoms. The signal beam will dress two of the three atomic states and give rise to energy shifts of

$$
\delta E=-\frac{1}{2} \Delta_{1} \pm\left(\frac{1}{4} \Delta_{1}^{2}+\Omega_{s}^{2}\right)^{1 / 2} .
$$


Note that in our notation positive detunings correspond to negative energy shifts. Its second effect will be to transfer all of the population to the third undressed state, thereby emptying the signal transition. If we label the signal transition levels $|1\rangle$ and $|2\rangle$ and the ground state of the probe transition $|0\rangle$, then in a zeroth-order approximation the solution for the atomic steady-state coherences will be $\sigma_{i j}=\delta_{i 0} \delta_{j 0}$. The inclusion of terms coming from the probe field yields nonzero atomic coherences for the probe transition $|0\rangle-|2\rangle$ and the twophoton transition between levels $|0\rangle-|1\rangle$. The atomic coherence $\sigma_{12}$ for the signal transition and all other population terms are of second order in the probe Rabi frequency. The first-order atomic coherence $\sigma_{02}$ governs the atomic response, i.e., the absorptive and refractive behavior of the atoms, to the probe beam. It is found to be

$$
\begin{aligned}
\sigma_{02}^{(1)} & =\Omega_{p}(a+i n) \\
& =\Omega_{p} \Delta\left\{\frac{\Delta \gamma}{|M|^{2}}+i \frac{\left|\Omega_{s}\right|^{2}-\Delta \Delta_{2}}{|M|^{2}}\right\},
\end{aligned}
$$

where the two-photon detuning $\Delta$ is given by $\Delta=\Delta_{2}-\Delta_{1}$ and $M=D+i \Delta \gamma$, with

$$
\begin{aligned}
D= & \left|\Omega_{s}\right|^{2}-\Delta_{2} \\
= & \left\{\Delta_{1} / 2-\Delta_{2}+\left[\Omega_{s}^{2}+\left(\Delta_{1} / 2\right)^{2}\right]^{1 / 2}\right\} \\
& \times\left\{\Delta_{2}-\Delta_{1} / 2+\left[\Omega_{s}^{2}+\left(\Delta_{1} / 2\right)^{2}\right]^{1 / 2}\right\} .
\end{aligned}
$$

$D$ is the product of the frequency differences between the probe input frequency $\omega_{p}^{\text {in }}$ (level $|u\rangle$ in Fig. 2) and the transition frequencies between level $|0\rangle$ and the lightshifted levels $|+\rangle,|-\rangle$ of the excited state $|2\rangle$.

In Fig. 3 we plot the spectral absorption coefficient $a$ and the nonlinear phase shift $n$ versus the atomic probe detuning $\Delta_{2}$. The two peaks correspond to maximum absorption at the light-shifted levels.

We realize that absorption is small in between the peaks, especially around $\Delta_{1}=\Delta_{2}$. For the $\Lambda$ atom, this two-photon resonance corresponds to coherent population trapping. In this probe-frequency range, probe absorption is very small and quadratic, while the nonlinear phase shift $n$ exhibits linear growth, as can be seen from Fig. 3.

In order to obtain good QND coupling, one has to keep absorption low on the probed transition and still retain a reasonably large nonlinear frequency shift for the probe beam. This can be obtained by tuning the probe beam to the vicinity of the light-shifted level so that we can operate in the wings of the absorption line. This may be achieved by requiring that

$$
|\eta| \ll<1
$$

where

$$
\eta=\frac{a}{n}=\frac{\Delta \gamma}{D}
$$

Throughout the rest of this paper, it will prove to be convenient to introduce quadrature operators; hence, we define

$$
\begin{aligned}
& X_{j}=\frac{\alpha_{j}^{*}}{\left|\alpha_{j}\right|} \delta a_{j}+\frac{\alpha_{j}}{\left|\alpha_{j}\right|} \delta a_{j}^{\dagger}, \\
& Y_{j}=-i\left(\frac{\alpha_{j}^{*}}{\left|\alpha_{j}\right|} \delta a_{j}-\frac{\alpha_{j}}{\left|\alpha_{j}\right|} \delta a_{j}^{\dagger}\right),
\end{aligned}
$$

with $j=s, p$. The annihilation and creation operators for the fluctuations in the two light fields are denoted by $\delta a_{j}$ and $\delta a_{j}^{\dagger}$, whereas $\alpha_{k}$ stands for the macroscopic mean complex amplitudes of the two laser modes. Our definition is especially convenient if the output beams are measured with respect to the phases of the input beams. The atomic-noise quadrature operators $X_{j}^{a}, Y_{k}^{a}$ are defined analogously to the field quadratures and used to calculate the atomic-noise correlation matrix [cf. Eq. (12)].

The quantum stochastic properties of the probe and signal field depend on the linear atomic response to the fields and on atomic-noise terms describing spontaneous emission. In the frequency domain, this can be formulated concisely in terms of susceptibility coefficients which establish a linear relation between fluctuations of the atomic polarizations and light-field quantum fluctuations [14]. If we denote the atomic response to the field fluctuations by $R$, then we find the simple relation

$$
\mathbf{R}(\omega)=\chi(\omega)\left[\begin{array}{c}
X_{s}(\omega) \\
Y_{s}(\omega) \\
X_{p}(\omega) \\
Y_{p}(\omega)
\end{array}\right]+\mathbf{B}_{a}(\omega)
$$

where the atomic-noise quadrature operators are arranged in the vector $\mathbf{B}_{a}$. We will outline this in greater detail in Sec. VI. One may obtain analytical expressions for the susceptibility matrix $\chi$ and the atomic-noise correlation matrix $\mathbf{G}_{a}=\left\langle\mathbf{B}_{a} \mathbf{B}_{a}^{T}\right\rangle$ under the following restrictions.

(1) We only calculate the zero-frequency component.

(2) We introduce an ordering scheme such that $\epsilon=\left|\Omega_{p} / \Omega_{s}\right|$ and $\eta$, as defined in Eq. (9), are of the same order of magnitude and only lowest-order terms in the product of both parameters are considered.

Then we find for $\Lambda$ - and cascade-model atoms that

$$
\begin{aligned}
\chi & =\left[\begin{array}{cccc}
0 & 0 & 0 & 0 \\
0 & 0 & q+b & 0 \\
0 & 0 & a & n \\
q & 0 & -n & a
\end{array}\right] \\
\mathbf{G}_{a} & =\left[\begin{array}{llcc}
0 & 0 & 0 & 0 \\
0 & p & b & -i b \\
0 & b & 2 a & 2 i a \\
0 & i b & -2 i a & 2 a
\end{array}\right]
\end{aligned}
$$

where the nonzero coefficients in the case of $\Lambda$ atoms are defined as 


$$
\begin{aligned}
& \left.n=-C_{p} \frac{\Delta \Gamma_{2}}{D} \text { (probe frequency shift in units of } \Gamma_{p}\right) \\
& a=-\eta n=C_{p} \frac{\gamma \Delta^{2} \Gamma_{2}}{D^{2}} \text { (probe absorption) } \\
& q=-2 \sqrt{C_{s} C_{p}} \frac{\Omega_{p} \Omega_{s}}{D} \frac{\Delta \sqrt{\Gamma_{1} \Gamma_{2}}}{D} \text { (QND coefficient) } \\
& b=-2 \sqrt{C_{s} C_{p}} \frac{\Omega_{p}}{\Omega_{s}} \frac{\Gamma_{1}}{\Gamma_{2}} \frac{\Delta_{1} \Delta^{2} \sqrt{\Gamma_{1} \Gamma_{2}}}{D^{2}} \text { (correlated noise coefficient) } \\
& p=C_{s} \frac{\Omega_{p}^{2}}{\Omega_{s}^{2}} \frac{\Gamma_{1}^{2}}{\Gamma_{2}^{2}} 4 \Delta^{2} \frac{\left(\Gamma_{2} / \gamma\right) \Omega_{s}^{2}+\Delta_{1}^{2}}{D^{2}} \text { (atomic signal-phase excess noise) }
\end{aligned}
$$

where $C_{s}$ and $C_{p}$ are the cooperativities for each field and are defined in Sec. VI. Note that $a$ and $n$ have a slightly different expression compared with $a$ and $n$ of Eq. (8). The frequency shift $n$ is only listed for completeness since it can always be compensated by detuning the cavity a corresponding amount into the opposite direction, thereby maximizing the interaction between the two light fields.

Using Eq. (12) and standard input-output relations as developed by Gardiner and Collett [11], we are able to evaluate the zero-frequency component of the three spectral correlation coefficients characterizing [12] a QND device. For completeness, we also give the equivalent input noises as introduced in Ref. [13]; in our case, these are related to the correlation coefficients by $C^{2}=\left(1+N^{\mathrm{eq}}\right)^{-1}$. We obtain, for the zero-frequency component of the spectral-measurement correlation coefficient, characterizing the ability of the scheme as a measurement device,

$$
\begin{aligned}
C^{2}\left(X_{s}^{\text {in }} Y_{p}^{\text {out }}\right) & =\frac{\left|\left\langle X_{s}^{\text {in }} Y_{p}^{\text {out }}\right\rangle_{\text {sym }}\right|^{2}}{\left\langle\left(X_{s}^{\text {in }}\right)^{2}\right\rangle\left\langle\left(Y_{p}^{\text {out }}\right)^{2}\right\rangle} \\
& =\frac{4 q^{2}}{4 q^{2}+a^{2}+2 a+1}
\end{aligned}
$$

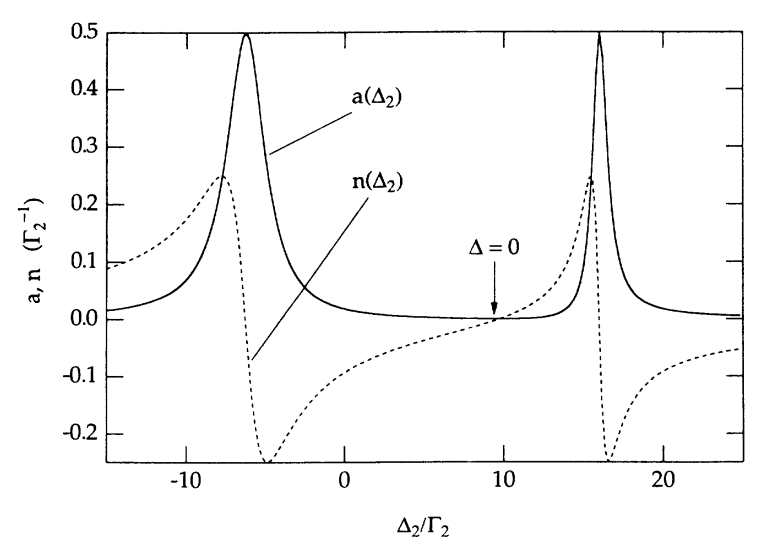

FIG. 3. Probe absorption $a$ and nonlinear phase shift $n$ as a function of the scaled atom-probe detuning $\Delta_{2} / \Gamma_{2}$. Zero absorption corresponds to the two-photon-resonance case $\Delta=0$. Note the quasilinear behavior of $n$ around $\Delta=0$, where absorption $\simeq \Delta^{2}$. The peaks occur at the light-shifted levels for $\Delta_{2}=-\delta E$ [cf. Eq. (7)], with a width of the size of $\gamma$. $\Delta_{1}=\Omega_{s}=10, \gamma=2$ in units of $\Gamma_{2}$. and

$$
N^{\mathrm{eq}}\left(X_{s}^{\text {in }} Y_{p}^{\text {out }}\right)=\frac{(a+1)^{2}}{4 q^{2}} .
$$

The correlation coefficient of the signal-output amplitude quadrature with its input counterpart gives information about degradation of the signal field in the cavity. It is found to be

$$
C^{2}\left(X_{s}^{\text {in }} X_{s}^{\text {out }}\right)=\frac{\left|\left\langle X_{s}^{\text {in }} X_{s}^{\text {out }}\right\rangle_{\text {sym }}\right|^{2}}{\left\langle\left(X_{s}^{\text {in }}\right)^{2}\right\rangle\left\langle\left(X_{s}^{\text {out }}\right)^{2}\right\rangle}=1 .
$$

In terms of equivalent input noises, this reads $N^{\mathrm{eq}}=0$. Finally, we find, for the conditional variance describing the state-preparation ability of the device,

$$
\begin{aligned}
V\left(X_{s}^{\text {out }} \mid Y_{p}^{\text {out }}\right) & =1-C^{2}\left(X_{s}^{\text {in }} Y_{p}^{\text {out }}\right) \\
& =\frac{(a+1)^{2}}{4 q^{2}+a^{2}+2 a+1} .
\end{aligned}
$$

From Eqs. (14) and (16), we realize that the requirement $q \gg \max (a, 1)$ is essential to obtain good QND correlations. Since $a$ and $q$ depend on the cooperativities $C_{p}$ and $C_{s}$, this requirement may be used to estimate the minimum cooperativities compatible with good QND performance.

We now calculate the degradation the signal phase experiences due to the measurement. The minimum degradation of the signal phase is obtained from the Heisenberg relation

$$
\begin{aligned}
V\left(Y_{s}^{\text {out }}\right) & =V\left(Y_{s}^{\text {out }} \mid Y_{p}^{\text {out }}\right) \\
& \geq \frac{1}{V\left(X_{s}^{\text {out }} \mid Y_{p}^{\text {out }}\right)}=1+\frac{4 q^{2}}{(1+a)^{2}},
\end{aligned}
$$

while we find

$$
V\left(Y_{s}^{\text {out }}\right)=1+2 p+4 q \frac{b+q}{1+a} .
$$

We realize that the signal phase has picked up some excess noise, which shows that after the measurement the signal plus probe system is no longer in a minimumuncertainty state. Note that the parametric model $[5,6]$ which predicts a minimum-uncertainty state, cannot account for terms proportional to the coefficients $b$ and $p$, which are entirely due to atomic noise.

In order to show that the QND effect is not symmetri- 
cal, we now swap signal and probe and consider a hypothetical QND measurement of the probe-amplitude quadrature by measuring the signal-phase quadrature. In the limit under consideration, the degradation of the probe amplitude is only due to linear losses, as shown by the correlation coefficient

$$
C^{2}\left(X_{p}^{\text {in }} X_{p}^{\text {out }}\right)=\left(\frac{1-a}{1+a}\right)^{2},
$$

which only depends on the probe-absorption coefficient $a$. Finally, the measurement correlation and variance are given by

$$
\begin{aligned}
& C^{2}\left(X_{p}^{\text {in }} Y_{s}^{\text {out }}\right)=4\left(\frac{b+q}{1+a}\right]^{2}\left[1+2 p+4 q \frac{b+q}{1+a}\right]^{-1} \\
& V\left(X_{s}^{\text {out }} \mid Y_{p}^{\text {out }}\right)=\frac{4 q^{2}}{(1+a)^{2}}\left[1+2 p+4 q \frac{b+q}{1+a}\right]^{-1}
\end{aligned}
$$

We find that in this reversed situation the positive atomic-phase excess noise $p$ and the correlated atomic noise $b$ lead to an overall degradation of the QND performances, which is confirmed by numerical solutions of the complete model. This demonstrates clearly that in contrast to the parametric model [6], the QND scheme studied here is not symmetrical with respect to the exchange of the signal and meter beams, as a result of additional contributions from atomic-noise terms.

\section{IV. $\Lambda$ CONFIGURATION-NUMERICAL RESULTS AND DISCUSSION}

In this section we will give a qualitative discussion of the results derived above and contrast them with the corresponding numerical solutions.

Since we are dealing with a complete model for the interaction between probe and signal beams - i.e., all internal atomic degrees of freedom are accounted for-it is not possible to derive compact analytical solutions for an arbitrary set of parameters. Fortunately, we are mainly interested in the case of signal Rabi frequencies much stronger than the probe Rabi frequencies, and with the further approximation of $\eta \ll<1$ [cf. Eq. (9)] it is possible to derive simple analytical results for the zero-frequency components of the spectral QND correlation coefficients. A comparison with numerical results shows an excellent overall agreement with the analytical approximations. We may use the analytical results to contemplate promising parameter regions and general qualitative features. An exact evaluation is eventually carried out by calculating the QND correlations numerically.

For all these plots, we are operating around the lightshifted level, which leads to the smaller two-photon detuning $\Delta$. The QND performances when working around the other light-shifted level are not as good, which can be understood by noting that the probe-absorption coefficient $a$ goes like $\Delta^{2}$, whereas the QND coefficient $q$ is proportional to $\Delta$ [cf. Eq. (13)].

It is our objective to achieve almost perfect QND performance, as characterized by the three QND coefficients in Eqs. (14)-(16) for small cooperativities $\left(\leq 10^{3}\right)$ and a broad band of probe input detunings $\Delta_{2}$. In Figs. 4 and 5

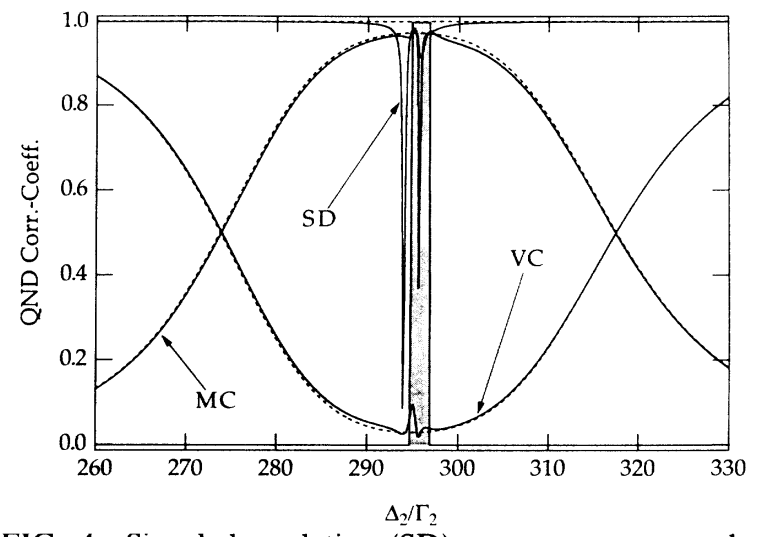

FIG. 4. Signal degradation (SD), measurement correlation (MC), and conditional variance (VC) at zero frequency as functions of the scaled atom-probe detuning. An instability occurs if the probe is almost resonant with the light-shifted level. Instabilities occur within the gray shaded area. The parameters are $\Omega_{p} / \Omega_{s}=0.005, \Delta_{1} / \Omega_{s}=0.8, \Gamma_{1} / \Gamma_{2}=1$, and $\Omega_{s}=200 \Gamma_{2}$. The cooperativities are $C_{s}=2000$ and $C_{p}=500$. Note the excellent agreement of the analytical (dashed curve) with the solid numerical solutions (solid curve). The analytical approximation to $D_{s}$ (dashed curve) is equal to 1 .

we plot the measurement correlation (MC) signal degradation (SD), and conditional variance (VC) for $\Omega_{s} / \Gamma_{2}=200$ and $\Delta_{1} / \Omega_{s}=0.8$ for different pairs of cooperativities $C_{s}=2000, C_{p}=500$ and $C_{s}=100, C_{p}=50$. For large cooperativities $\left(>10^{2}\right.$; cf. Fig. 4), we find indeed very good agreement, with peak height depending only weakly on the ratio of the cooperativities, but not on their size. For smaller cooperativities (cf. Fig. 5), however, this does not hold anymore and the analytical approximations fail to describe the peak height properly while still being a good approximation for the general shape. Also, a narrowing of the peaks with decreasing cooperativities is obvious. This feature eventually leads to a lower limit for the cooperativities since the most interesting region, the "flat" correlation maximum, is at some stage exclusively occupied by the instability region.

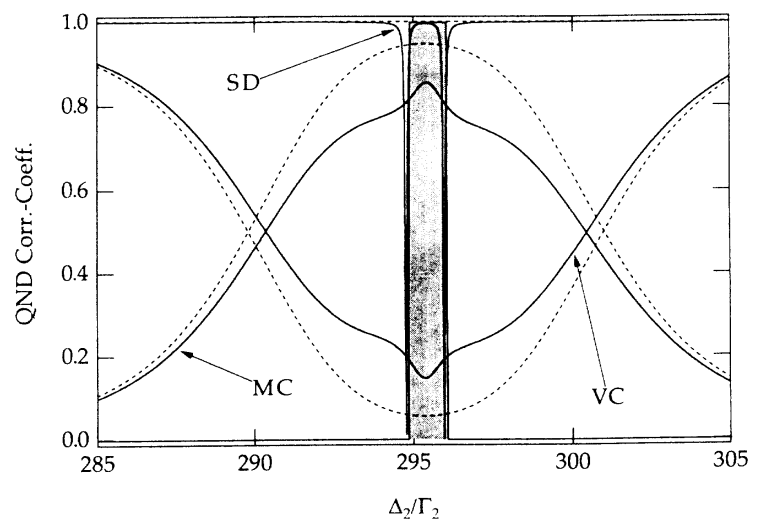

FIG. 5. Same as caption to Fig. 4 for very small cooperativities $C_{s}=100$ and $C_{p}=50$. Still reasonably good correlations can be achieved when the probe is tuned to the slopes of the peak in MC outside the central instability region. 
Operability of the device for too low cooperativities is then confined to the steep slopes of the curve, leading to unsatisfactory performance.

The disagreement between numerical and analytical solutions for probe detunings $\Delta_{2}$ around and on resonance with the light-shifted levels is due to the failure of the assumption that $\eta<1$ in those regions. In other words, approaching the light-shifted levels means changing from dispersive to absorptive operating conditions. Moreover, if the probe is almost resonant with the lightshifted levels, we encounter a small unstable region where the linearization done for the numerical computation (see Sec. VI) breaks down. This is due to the fact that tuning the coherent probe input to resonance with one of the Rabi-split levels leads to gain for sideband frequencies, which would eventually result in the buildup of small coherent sidebands on the signal transition. In order to describe the system properly in this situation by a linearized analysis, one would have to include sidebands explicitly into the model from the beginning. Note that the correlations can be significantly degraded as a result of absorption, even outside the instability region.

In Fig. 6 we plot the three spectral correlation coefficients as functions of the noise frequency $\omega$. We realize that signal degradation is virtually nonexistent, with SD less than $1 \%$ for the same parameters as in Fig. 4 with $\Delta_{2}=290 \Gamma_{2}$. The cavity decay rates are both equal to $4 \Gamma_{2}$; hence, except for SD, good QND performance can be expected mainly for frequencies smaller than one cavity linewidth. Note that at zero frequency $\mathrm{MC}$ is around $95 \%$ and $\mathrm{VC}$ is around $5 \%$. It is feasible to improve those even further at the expense of having to increase the cooperativities.

We shall now consider the effect of additional damping on the QND performances of this scheme. In its present form, our description of the $\Lambda$-model atom does not include damping terms for the atomic two-photon coherence $\sigma_{01}$ between the two low-lying levels $|0\rangle$ and $|1\rangle$. Such terms could arise from atomic collisions. While the cascade structure of the ladder model naturally provides

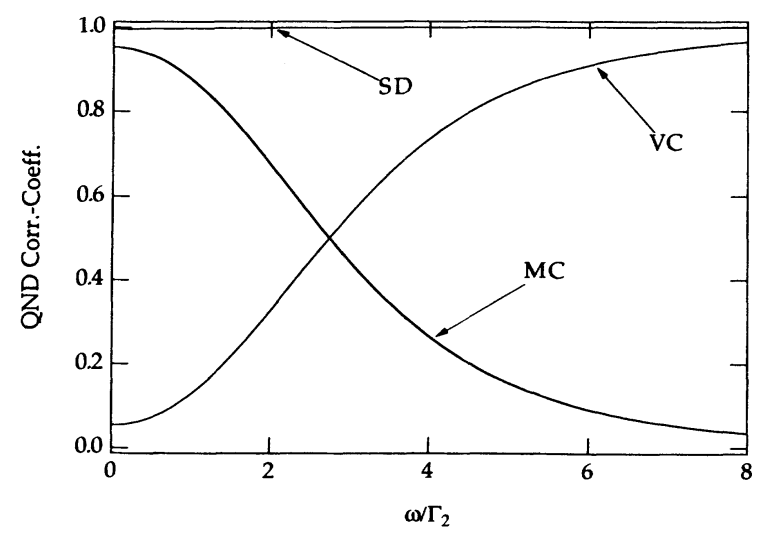

FIG. 6. Spectral correlation coefficients vs. frequency. Parameters are the same as in Fig. 4. $\Delta_{2}=290 \Gamma_{2}$. Note the almost perfect correlation between signal input and output due to the quasitransparency of the medium around the signal input frequency (atomic ghost transition). for damping of the two-photon coherence between the highest and lowest levels [cf. Fig. 1(b)] through spontaneous decays, no such effects are present in the $\Lambda$ model. Since relaxation between the two ground states may influence the atomic dynamics in many realistic situations, we will now investigate the effect of two different damping mechanisms.

Phase damping. Phase damping due to collisions will only increase relaxation for the atomic coherences while leaving the equations for the atomic populations unchanged. Numerical evaluation of the QND correlations in the parameter regions of interest, as discussed above, shows that this kind of damping does not seriously degrade correlation between signal and probe beams, provided the additional damping constant $\gamma_{p}$ remains reasonably small. This should, in fact, be guaranteed by employing rather small cooperativities and, hence, low atomic densities.

Ground-state relaxation. If we allow for population transfer between the two low-lying states, additional damping terms will affect all atomic operators. We find that large relaxation rates, i.e., of the same size as the other atomic rates, between states $|0\rangle$ and $|1\rangle$ deteriorate the QND performance substantially. Moreover, we find that less deterioration occurs if population transfer mainly occurs from the signal ground state to the probe ground state. The correlation coefficients most affected are MC and VC, while good signal input-output correlation is retained. As an example, let us assume equal rates for population transfer in both directions. The numerical solution for the same parameters as in Fig. 4 and equal ground-state relaxation rates of $\Gamma_{r}=0.1 \Gamma_{2}$ are compared in Fig. 7 with the analytical approximations valid for $\Gamma_{r}=0$. We realize that good performance can now only be achieved for a much smaller range of probe detunings $\Delta_{2}$. We also find that the conditional variance, and hence the signal amplitude, has picked up some excess noise of $\approx 0.05$ unit vacuum fluctuations resulting from the incoherent recycling of electrons back to the ground state of the signal transition. The fact that electrons may now

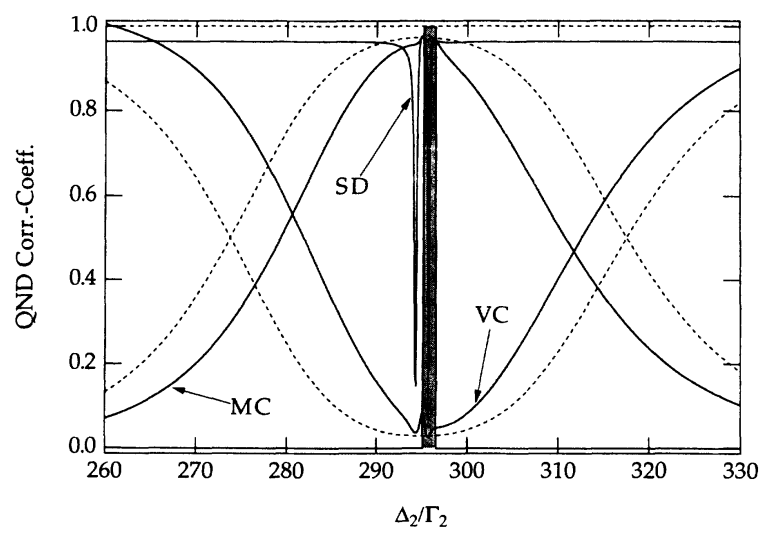

FIG. 7. Zero-frequency correlation coefficients for same parameters as in Fig. 4 with mutual ground-state relaxation at rate $\Gamma_{r}=0.1 \Gamma_{2}$. The dashed lines represent the analytical approximations for $\Gamma_{r}=0$. 
be transferred incoherently back to the signal ground state is the main cause for smaller QND correlations.

Strong relaxation between the ground states lets the $\Lambda$-model atom resemble the far simpler two-level Raman scheme, where it was shown that it is not possible to obtain QND performance as good as in the present proposal [12].

\section{CASCADE CONFIGURATION}

The cascade configuration used as a QND scheme has already been studied in several papers. In Ref. [6] analytical expressions were derived in the parametric limit, which cannot describe accurately the experimental results [5]. In the more complete calculation of Ref. [8], the two beams had a similar intensity and no analytical results for the QND criteria were derived. But here the range of parameters considered allows us to derive approximated analytical expressions, as we did for the $\Lambda$ system in the previous sections.

The strong signal beam (with Rabi frequency $\Omega_{s}$ ) is tuned around the upper transition (labeled 1). The amplitude of this signal is measured by the phase of the weak probe beam (with Rabi frequency $\Omega_{p}<<\Omega_{s}$ ) tuned around the lower transition (labeled 2). We work under the same restrictions as for the $\Lambda$ configuration; i.e., we calculate only the zero-frequency component up to the lowest significant order in the product of the two small parameters $\epsilon$ and $\eta$ defined in a similar manner as in Sec. IV (with the two-photon detuning now defined by $\left.\Delta=\Delta_{1}+\Delta_{2}\right)$. The susceptibility matrix $\chi$ and atomicnoise correlation matrix $\mathbf{G}_{a}$ have the same structure as for the $\Lambda$ scheme [cf. Eq. (12)]. However, the parameters $n, a, b, p, q$ involved have different expressions, which are the following:

$$
\begin{aligned}
& n=-C_{p} \frac{\Delta \Gamma_{2}}{D}, \\
& a=-\eta n=\frac{C_{p} \Gamma_{2}}{D^{2}}\left(\Gamma_{1} \Omega_{s}^{2}+\Gamma_{2} \Delta^{2}\right), \\
& q=-2 \sqrt{C_{s} C_{p}} \sqrt{\Gamma_{1} \Gamma_{2}} \frac{\Delta \Omega_{s} \Omega_{p}}{D^{2}}, \\
& b=-2 \frac{\sqrt{C_{s} C_{p}} \sqrt{\Gamma_{1} \Gamma_{2}} \frac{\Gamma_{1}^{2} \Delta_{1} \Omega_{s} \Omega_{p}^{3}}{D^{2}},}{p=C_{2}\left(\Omega_{s}^{2}+\Gamma_{1} \frac{\left.\Gamma_{1} \Omega_{1}^{2} / \gamma\right)}{\gamma^{2} \Omega_{2} D^{2}} \frac{\left(\Gamma_{2} \Omega_{s}^{2}+\Gamma_{1} \Delta_{1}^{2}\right)}{\left(\Omega_{s}^{2}+\Gamma_{1} \Delta_{1}^{2} / \gamma\right)^{2}} .\right.}
\end{aligned}
$$

Note that the probe-absorption coefficient $a$ contains now an extra term $\Gamma_{1} \Omega_{s}^{2}$ due to two-photon absorption. The consequence is that the probe absorption is never zero, whereas it was the case for the $\Lambda$ system at $\Delta=0$, where we had coherent population trapping (see Sec. III). This does not occur in the cascade system: At the two-photon resonance $\Delta=0$, only the dispersion goes to zero. Finally, it is to be noted that the QND coefficient $q$ has exactly the same expression as in the $\Lambda$ configuration.

The three QND correlation coefficients have the same appearance in terms of $a, b, p, q$ as in Eqs. (14)-(16).
Within this approximation there is no signal degradation and the measurement correlation coefficient $C^{2}\left(X_{s}^{\text {in }} Y_{p}^{\text {out }}\right)$ is related to the conditional variance $V\left(X_{s}^{\text {out }} \mid Y_{p}^{\text {out }}\right)$, describing the state-preparation ability of the device, by $V\left(X_{s}^{\text {out }} \mid Y_{p}^{\text {out }}\right)=1-C^{2}\left(X_{s}^{\text {in }} Y_{p}^{\text {out }}\right)$. So, in order to compare the QND properties of the cascade configuration with those of the $\Lambda$ scheme within this approximation, we just have to consider the expression of the measurement coefficient. This coefficient only involves $a$ and $q$, and only the probe-absorption $a$ has a different expression; we should therefore focus on this extra term.

If we take exactly the same parameters as in Fig. 4, the results are slightly degraded with respect to the $\Lambda$ scheme (0.93 instead of 0.96 for the measurement coefficient). But in order to get closer to the QND performances of the $\Lambda$ scheme, one can decrease the extra term $\Gamma_{1} \Omega_{s}^{2}$ of the probe absorption by taking a smaller $\Gamma_{1}$ [14]. We tune the probe frequency around the light-shifted level of the intermediate level that is the farthest away from the bare atomic level as we did in Fig. 4. We then obtain (cf. Fig. 8) similar QND performances as with the $\Lambda$ configuration. Note that we find the same disagreement of the analytical approximated results and the numerical results with this very narrow double-peaked structure as for the $\Lambda$ system.

This ladder scheme using a ghost transition gives definitely better results than the ladder scheme treated in Ref. [8] with two fields of comparable magnitude and with exactly the same numerical method. The measurement coefficient and signal degradation coefficient were both of about 0.97 , which is comparable to the present results, but the cooperativities were about 50 times larger than those used here. From an experimental point of view, the use of smaller cooperativities is more convenient. It indeed allows a reduction of the density of the beam, which diminishes the number of collisions, and/or operation with a better collimated beam, which narrows the Doppler width.

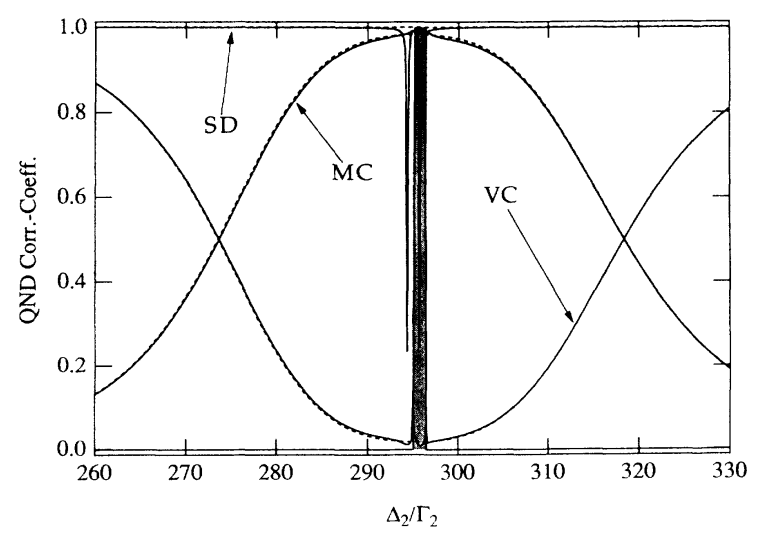

FIG. 8. Zero-frequency correlation coefficients for the ladder scheme vs scaled atom-probe detuning $\Delta_{2} / \Gamma_{2}$. The parameters are the same as in Fig. 4 except that the decay rate of the upper transition is decreased by a factor of 4 . The dashed lines represent the analytical approximated result. The gray area is the unstable region. 


\section{MATHEMATICAL MODEL}

This part of the paper is dedicated to a more thorough theoretical description of the interaction of two laserlight modes, labeled signal and probe, in the presence of $N$ three-level atoms inside an optical cavity. We assume we can model the coherent input light beams into the cavity by decomposing them into a classical coherent part $E_{j}(t)=E_{j} \exp \left(-i \omega_{j}^{\text {in }} t\right)$ and a fluctuation term $\int_{v \approx \omega_{j}^{\text {in }}} d v B_{j}(v)$ that is broadband around the input center frequency $\omega_{j}^{\text {in }}$, with $j=s, p$. The empty cavity is assumed to be resonant at frequencies $\omega_{s}$ and $\omega_{p}$, which are supposed to be equal to the input frequencies of the coherent light. The light fields inside the cavity are modeled by single-mode operators $A_{j}$ for those resonance frequencies. Because of the presence of atoms in the cavity, the resonance frequencies of the cavity will change by a certain amount, as shown in Sec. III. We will assume that it is always possible to compensate for those frequency shifts by either changing the length of the cavity or detuning the input beams.

The atoms are modeled by closed three-level systems where the two dipole-allowed transitions enabled radiative transitions from the two nondegenerate ground states to a common excited state. The two ground states are la- beled $|0\rangle$ and $|1\rangle$, the excited state $|2\rangle$. The two allowed atomic transitions interact with incoherent fields $B_{j}(t)$, $j=\{1,2\}$, leading to spontaneous decay with decay constants $\Gamma_{1}$ and $\Gamma_{2}$. Other relaxation mechanisms leading to incoherent relaxation between the two low-lying states or additional dephasing for the coherence between those same levels as discussed in Sec. III can be included similarly. The quantized intracavity fields $A_{s}$ and $A_{p}$ are perturbed by their interaction with the cavity reservoirs $B_{s}(t)$ and $B_{p}(t)$; the empty cavity damping rates are denoted by $\Gamma_{s}$ and $\Gamma_{p}$, respectively. All noise operators are modeled by quantum white noise (QWN).

\section{A. Hamiltonian}

The theoretical description of the above system is given by the explicitly time-dependent Hamiltonian $\mathscr{H}(t)$ :

$$
\mathscr{H}(t)=\mathscr{H}_{\mathrm{sys}}+\mathscr{H}_{\mathrm{res}}+\mathscr{H}_{\mathrm{int}},
$$

consisting of three parts. The system Hamiltonian describes the interaction of $N$ three-level atoms with two quantized cavity light modes $A_{s}$ and $A_{p}$ and their interaction with two classical coherent inputs into the cavity denoted by $E_{s}$ and $E_{p}$, respectively:

$$
\begin{aligned}
\mathscr{H}_{\text {sys }}= & \hbar \sum_{\mu=1}^{N_{a}}\left\{\omega_{1} \sigma_{11}^{\mu}+\omega_{2} \sigma_{22}^{\mu}+\omega_{s} \frac{A_{s}^{\dagger} A_{s}}{N}+\omega_{p} \frac{A_{p}^{\dagger} A_{p}}{N}-\left(g_{s} \sigma_{20}^{\mu} A_{s}+\text { H.c. }\right)-\left(g_{p} \sigma_{21}^{\mu} A_{p}+\text { H.c. }\right)\right\} \\
& +i \hbar\left\{\left[A_{s}^{\dagger} E_{s}(t)-\text { H.c. }\right]+\left[A_{p}^{\dagger} E_{p}(t)-\text { H.c. }\right]\right\} .
\end{aligned}
$$

The reservoir Hamiltonian describes the four baths responsible for cavity damping of the two electromagnetic modes and atomic spontaneous-decay processes:

$$
\mathscr{H}_{\mathrm{res}}=\hbar\left\{\int_{v \approx \omega_{2}-\omega_{1}} d v v B_{1}^{\dagger}(v) B_{1}(v)+\int_{v \approx \omega_{2}} d v v B_{2}^{\dagger}(v) B_{2}(v)+\int_{v \approx \omega_{s}} d v v B_{s}^{\dagger}(v) B_{s}(v)+\int_{v \approx \omega_{p}} d v v B_{p}^{\dagger}(v) B_{p}(v)\right\} .
$$

The interaction between the system variables and various baths is described by the interaction Hamiltonian

$$
\begin{aligned}
\mathscr{H}_{\mathrm{int}}= & -\hbar \sum_{\mu=1}^{N_{a}}\left\{\left[g_{1}^{\dagger} \int_{v \approx \omega_{2}-\omega_{1}} d v B_{1}^{\dagger}(v) \sigma_{12}^{\mu}+\text { H.c. }\right)+\left[g_{2}^{\dagger} \int_{v \approx \omega_{2}} d v B_{2}^{\dagger}(v) \sigma_{02}^{\mu}+\text { H.c. }\right)\right\} \\
& +i\left(\frac{\Gamma_{s}}{\pi}\right)^{1 / 2}\left[\int_{v \approx \omega_{s}^{\mathrm{in}}} d v B_{s}^{\dagger}(v) A_{s}-\text { H.c. }\right)+i\left(\frac{\Gamma_{p}}{\pi}\right]^{1 / 2}\left(\int_{v \approx \omega_{p}^{\mathrm{in}}} d v B_{p}^{\dagger}(v) A_{p}-\text { H.c. }\right) .
\end{aligned}
$$

From this Hamiltonian we may now derive Ito-quantum stochastic differential equations (QSDE) [15] for the 12 system operators in $\mathscr{H}_{\text {sys. }}$. This approach is equivalent to the derivation of a master equation for the system-density operator.

\section{B. Linearization}

In order to simplify the analysis, we assume that we may linearize the set of Ito equations around the nonzero steady-state expectation values of a suitable set of system operators. The linearization per se implies the existence of a small quantity which serves as a series-expansion coefficient in an asymptotic power series. According to Gardiner [15], this small parameter is determined to be
$1 / \sqrt{N}$ after having introduced an appropriate scaling. We will also make the necessary assumption that the numbers of photons in each cavity mode are such that $N_{j}^{1 / 2} \gg 1$, for $j=p, s$. The introduction of the following abbreviations is found to be useful:

$$
\begin{aligned}
& \sigma_{i j}=\sum_{\mu=1}^{N_{a}} \frac{\sigma_{i j}^{\mu}}{N}, \\
& D_{2}=\sigma_{22}-\sigma_{00}, \\
& D_{1}=\sigma_{22}-\sigma_{11}, \\
& \Omega_{k}=i g_{k} A_{k},
\end{aligned}
$$




$$
C_{k}=\frac{\left|g_{k}\right|^{2} N}{\Gamma_{l} \Gamma_{k}} \text { for }(k, l)=(s, 1),(p, 2),
$$

where $C_{s}$ and $C_{p}$ are the cooperativity parameters for signal and probe fields, respectively.

For a large number of atoms, $N$, in the cavity, any system operator $S$ can be expanded into its semiclassical mean plus a fluctuation term proportional to $1 / \sqrt{N}$ :

$$
S \approx\langle S\rangle_{\mathrm{secl}}+\frac{1}{\sqrt{N}} S^{1}+o\left(\frac{1}{N}\right)
$$

This approximation is a reasonable one as long as the correlations of the first-order terms remain sufficiently smaller than $N$. Assuming this to be the case, i.e., not operating too close to a critical point, we find the zerothorder or semiclassical equations for the atoms in a frame rotating at the frequencies $\omega_{s}^{\text {in }}$ of the signal laser input and $\omega_{p}^{\text {in }}$ of the probe laser input into the cavity to be

$$
\partial_{t}\langle\Sigma\rangle=\mathbf{M}_{a}\langle\Sigma\rangle+\mathbf{I} \text {. }
$$

The atomic operators are arranged in the vector-valued operator

$$
\Sigma=\left[\sigma_{01}, \sigma_{10}, \sigma_{12}, \sigma_{21}, \sigma_{02}, \sigma_{20}, D_{2}, D_{1}\right]^{T} .
$$

The matrix-valued time-evolution generator $\mathbf{M}_{a}$ and the inhomogeneous $c$-number vector $\mathbf{I}$ are given by

$$
\mathbf{M}_{a}=\left[\begin{array}{cccccccc}
-\gamma_{01} & 0 & 0 & -\Omega_{p} & -\Omega_{s}^{*} & 0 & 0 & 0 \\
0 & -\gamma_{01}^{*} & -\Omega_{p}^{*} & 0 & 0 & -\Omega_{s} & 0 & 0 \\
0 & \Omega_{p} & -\gamma_{12} & 0 & 0 & 0 & 0 & -\Omega_{s} \\
\Omega_{p}^{*} & 0 & 0 & -\gamma_{12}^{*} & 0 & 0 & 0 & -\Omega_{s}^{*} \\
\Omega_{s} & 0 & 0 & 0 & -\gamma_{02} & 0 & -\Omega_{p} & 0 \\
0 & \Omega_{s}^{*} & 0 & 0 & 0 & -\gamma_{02}^{*} & -\Omega_{p}^{*} & 0 \\
0 & 0 & \Omega_{s}^{*} & \Omega_{s} & 2 \Omega_{p}^{*} & 2 \Omega_{p} & -\gamma_{2}^{2} & -\gamma_{2}^{1} \\
0 & 0 & 2 \Omega_{s}^{*} & 2 \Omega_{s} & \Omega_{p}^{*} & \Omega_{p}^{*} & -\gamma_{1}^{2} & -\gamma_{1}^{1}
\end{array}\right]
$$

and

$$
\mathbf{I}=-\left[0,0,0,0,0,0, \frac{2}{3}\left(2 \Gamma_{2}+\Gamma_{1}\right), \frac{2}{3}\left(2 \Gamma_{1}+\Gamma_{2}\right)\right]^{T} .
$$

The complex decay-detuning coefficients $\gamma_{i j}$ and the real $\gamma_{i}^{j}$ are functions of the natural atomic linewidths $\Gamma_{1}, \Gamma_{2}$. We find, for the decay constants,

$$
\begin{aligned}
& \gamma_{01}=i \Delta, \\
& \gamma_{12}=\Gamma_{1}+\Gamma_{2}+i \Delta_{1}, \\
& \gamma_{02}=\Gamma_{1}+\Gamma_{2}+i \Delta_{2}, \\
& \gamma_{2}^{2}=\frac{2}{3}\left(\Gamma_{1}+2 \Gamma_{2}\right), \\
& \gamma_{2}^{1}=\gamma_{2}^{2}, \\
& \gamma_{1}^{2}=\frac{2}{3}\left(2 \Gamma_{1}+\Gamma_{2}\right), \\
& \gamma_{1}^{1}=\gamma_{1}^{2}
\end{aligned}
$$

and for the detunings,

$$
\begin{aligned}
& \Delta_{1}=\omega_{2}-\omega_{1}-\omega_{s}^{\text {in }}, \\
& \Delta_{2}=\omega_{2}-\omega_{p}^{\text {in }}, \\
& \Delta=\Delta_{2}-\Delta_{1} .
\end{aligned}
$$

In order to simplify typing, we assume we may leave out brackets denoting expectation values. The fluctuation-correction terms for system operators are denoted by carets to distinguish them clearly from their semiclassical values. For the signal-field fluctuation operator $\hat{\Omega}_{s}$ we find the following quantum stochastic differential equation:

$$
\begin{aligned}
d \hat{\Omega}_{s}(t)= & -\left(\Gamma_{s}+i \Delta_{s}\right) \hat{\Omega}_{s}(t) d t-C_{s} \Gamma_{s} \Gamma_{1} \hat{\sigma}_{12} d t \\
& -\sqrt{2 \Gamma_{1} C_{s}} \Gamma_{s} d B_{s}(t),
\end{aligned}
$$

where $\left\langle d B_{s}(t) d B_{s}^{\dagger}(t)\right\rangle=d t$ and all other combinations are zero for ordinary vacuum inputs. The detuning $\Delta_{s}$ is defined analogously to the atom-input-field detunings. The equations for the other laser-field fluctuation operators may now be obtained by simple index manipulations from Eq. (32). Since any correlation between probe and signal laser fields can only arise from their common interaction with the atoms, we will have to solve the complete set of equations for all 12 atomic and field fluctuation operators to evaluate any kind of correlation function between signal and probe fields. Although the procedure to calculate those correlations numerically is straightforward, it is not suitable to obtain analytical results.

An alternative procedure useful for analytical approximations is to derive the susceptibility matrix $\chi(\omega)$ introduced in Sec. III. This can be done from the Ito QSDE for the 12 fluctuation operators, using projection techniques in atom-and-field subspaces and Fourier transforms. A more straightforward way is described in Ref. [10]: The atomic response is calculated first as if the atoms were in free space, using the quantum regression theorem. This yields directly $\chi(\omega)$ and $\mathbf{G}_{a}(\omega)$, which are then inserted in the standard input-output formalism to 
get the field correlation functions. Both procedures actually yield exactly the same results. Finally, in order to obtain the compact results listed in Eqs. (12) and (21), it is necessary to carry out a perturbative expansion in the parameters $\epsilon$ and $\eta$. This is done the most convenient way using computer software capable of carrying out formal algebraic computations.

\section{CONCLUSIONS}

We have analyzed in this paper a scheme for the realization of QND measurement using ghost transitions in three-level atoms, either in a $\Lambda$ configuration or in a cascade configuration. The principle is that we manage to transfer most of the atomic population into one level (one of the ground levels for the $\Lambda$ configuration and the ground level for the cascade scheme), which leaves the other transition almost empty. The strong signal beam tuned around this ghost transition is therefore hardly absorbed, while the weak meter beam probes the lightshifted levels induced by the signal beam. We have ob- tained approximated analytical results which are in very good agreement with the numerical results. For the range of parameters considered in this paper, we have not found essential differences in the QND performances of the $\Lambda$ and cascade configurations. The QND performances predicted by this scheme are almost perfect and rather realistic from an experimental point of view. They might, therefore, allow important improvements in the experimental realization of QND measurements.

\section{ACKNOWLEDGMENTS}

This work was supported by The University of Auckland Research Committee, The New Zealand Vice Chancellors Committee, the New Zealand Lottery Grants Board, and IBM, New Zealand. J.P.P. was supported by the French Ministry of Foreign Affairs. P.G. thanks the University of Auckland for its hospitality during his stay in New Zealand, where the main part of this work was carried out. K.M.G. thanks the Institut d'Optique, which supported this work.
*Permanent address: Physics Department, University of Auckland, Private Bag 92019, Auckland, New Zealand.

[1] G. J. Milburn and D. F. Walls, Phys. Rev. A 28, 2055 (1983).

[2] N. Imoto, H. A. Haus, and Y. Yamamoto, Phys. Rev. A 32, 2287 (1985).

[3] B. Yurke, J. Opt. Soc. Am. B 2, 732 (1985).

[4] M. D. Levenson, R. M. Shelby, M. Reid, and D. F. Walls, Phys. Rev. Lett. 57, 2473 (1986).

[5] P. Grangier, J. F. Roch, and G. Roger, Phys. Rev. Lett. 66, 1418 (1991).

[6] P. Grangier, J. F. Roch, and S. Reynaud, Opt. Commun. 72, 387 (1989).

[7] C. A. Blockley and D. F. Walls, Opt. Commun. 79, 241 (1990).

[8] J. Ph. Poizat, M. J. Collett, and D. F. Walls, Phys. Rev. A
45, 5171 (1992).

[9] H. A. Bachor and P. T. H. Fisk, Appl. Phys. B 49, 291 (1989).

[10] M. J. Holland, M. J. Collett, D. F. Walls, and M. D. Levenson, Phys. Rev. A 42, 2995 (1990).

[11] P. Grangier, J. M. Courty, and S. Reynaud, Opt. Commun. (to be published).

[12] J.-M. Courty, P. Grangier, L. Hilico, and S. Reynaud, Opt. Commun. 83, 251 (1991).

[13] C. W. Gardiner and M. J. Collett, Phys. Rev. A 31, 3761 (1985).

[14] Note that in most atoms the decay rate of the upper transition is smaller than the one of the lower transition. The decrease of $\Gamma_{1}$ is therefore rather realistic.

[15] C. W. Gardiner, Quantum Noise (Springer-Verlag, Berlin, 1991), Chaps. 5 and 9. 


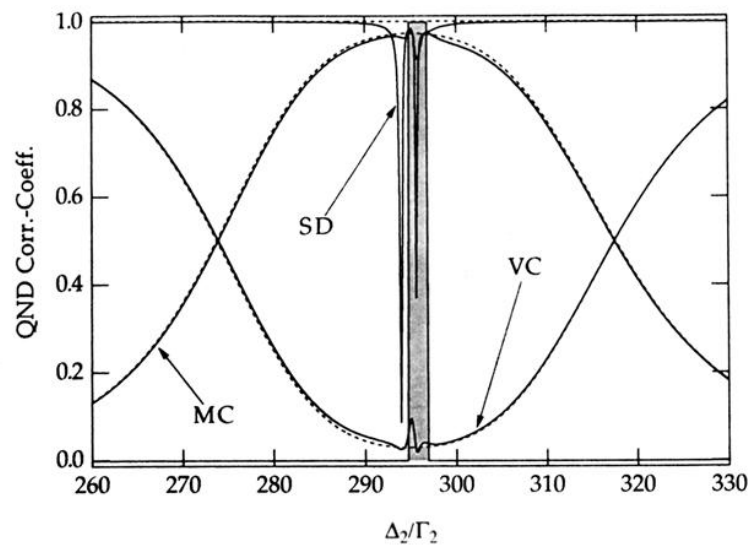

FIG. 4. Signal degradation (SD), measurement correlation (MC), and conditional variance (VC) at zero frequency as functions of the scaled atom-probe detuning. An instability occurs if the probe is almost resonant with the light-shifted level. Instabilities occur within the gray shaded area. The parameters are $\Omega_{p} / \Omega_{s}=0.005, \Delta_{1} / \Omega_{s}=0.8, \Gamma_{1} / \Gamma_{2}=1$, and $\Omega_{s}=200 \Gamma_{2}$. The cooperativities are $C_{s}=2000$ and $C_{p}=500$. Note the excellent agreement of the analytical (dashed curve) with the solid numerical solutions (solid curve). The analytical approximation to $D_{s}$ (dashed curve) is equal to 1 . 


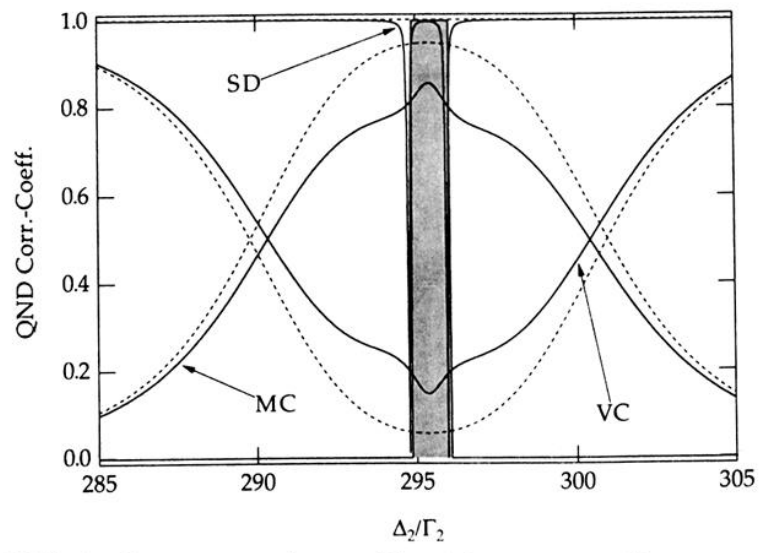

FIG. 5. Same as caption to Fig. 4 for very small cooperativities $C_{s}=100$ and $C_{p}=50$. Still reasonably good correlations can be achieved when the probe is tuned to the slopes of the peak in MC outside the central instability region. 


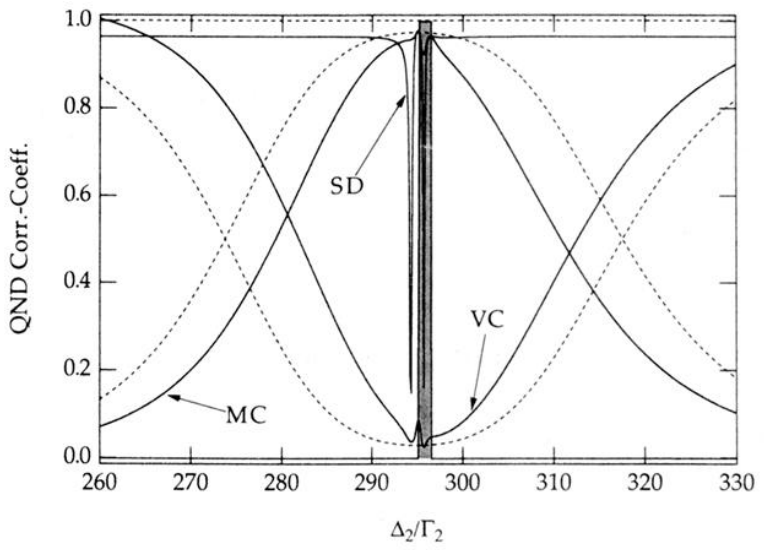

FIG. 7. Zero-frequency correlation coefficients for same parameters as in Fig. 4 with mutual ground-state relaxation at rate $\Gamma_{r}=0.1 \Gamma_{2}$. The dashed lines represent the analytical approximations for $\Gamma_{r}=0$. 


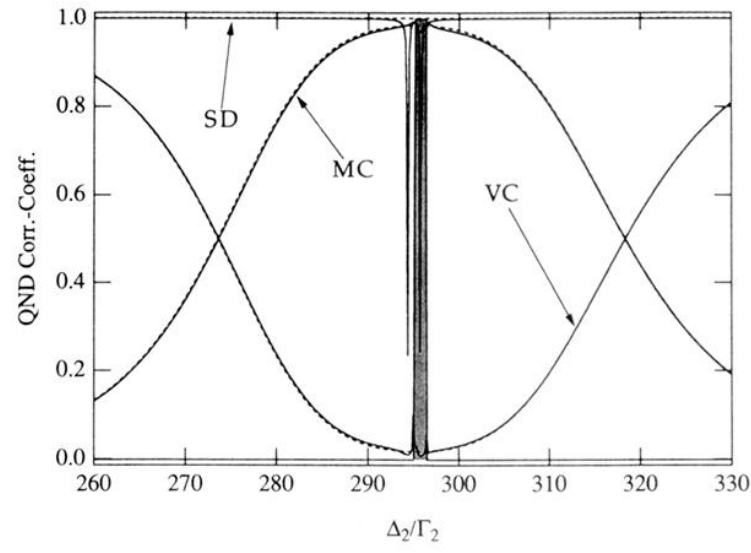

FIG. 8. Zero-frequency correlation coefficients for the ladder scheme vs scaled atom-probe detuning $\Delta_{2} / \Gamma_{2}$. The parameters are the same as in Fig. 4 except that the decay rate of the upper transition is decreased by a factor of 4 . The dashed lines represent the analytical approximated result. The gray area is the unstable region. 